\title{
Application of Wavelet Transform Modulus Maxima in Raman Distributed Temperature Sensors
}

\author{
Zongliang WANG, Jun CHANG*, Sasa ZHANG, Sha LUO, Cuanwu JIA, Boning SUN, \\ Shuo JIANG, Yongning LIU, Xiaohui LIU, Guangping LV, and Zhi LIU
}

School of Information Science and Engineering and Shandong Provincial Key Laboratory of Laser Technology and Application, Shandong University, Jinan, 250100, China

*Corresponding author: Jun CHANGＥ-mail: changjun@sdu.edu.cn

\begin{abstract}
We proposed and demonstrated a wavelet transform modulus maxima (WTMM) de-noising method to decrease the temperature error. In this scheme, the composition scale was determined simply by the WTMM amplitude variation with the growth of the decomposition scale at $30{ }^{\circ} \mathrm{C}$, and the signal WTMM was obtained by the wavelet decomposition modulus on every decomposition scale based on the modulus propagating difference between the signal and noise. Then, we reconstructed the signal using the signal WTMM. Experimental results show that the proposed method is effective for de-noising, allowing for a temperature error decrease of about $1{ }^{\circ} \mathrm{C}$ at $40{ }^{\circ} \mathrm{C}$ and $50{ }^{\circ} \mathrm{C}$ comparing to the original data.
\end{abstract}

Keywords: Distributed temperature sensor, Raman, wavelet transform modulus maxima

Citation: Zongliang WANG, Jun CHANG, Sasa ZHANG, Sha LUO, Cuanwu JIA, Boning SUN, et al., "Application of Wavelet Transform Modulus Maxima in Raman Distributed Temperature Sensors," Photonic Sensors, 2014, 4(2): 142-146.

\section{Introduction}

The Raman distributed optical fiber sensors can realize continuous temperature measurement based on the Raman backscattering. Owing to the well-known advantages of the resistance to electromagnetic interference and extended distance, the sensors have been the main research topic in the past few years [1-4].

Due to the immunity to electromagnetic interference (EMI) and radiation, they can offer the capability of easy handling to be applied in the harsh environment such as fire detection, power cable monitoring, and leakage detection. Raman distributed temperature sensors (RDTS) determine the fiber position using the optical time domain reflectometry (OTDR) technique. A short laser pulse is launched into the sensing fiber, and various kinds of scattering (such as Rayleigh scattering, Raman scattering, and Brillouin scattering.) are caused during the propagation of light [5]. In particular, the Rayleigh scattering is unaffected by the temperature, and Stokes scattering is also slightly temperaturedependent, while the anti-Stokes scattering is sensitive to the temperature. For the conventional RDTS system, the fiber temperature is usually obtained by the ratio of anti-Stokes to Stokes light. According to Raman spectroscopy, the light is about $30 \mathrm{~dB}$ weaker than the Rayleigh backscattered light, which is very difficult to be detected [6]. Meanwhile, these factors what the inhomogeneous medium and backscattering effect cause in the system will generate the optical noise, and the data acquisition

Received: 6 January 2014 / Revised version: 21 February 2014

(C) The Authors(s) 2014. This article is published with open access at Springerlink.com

DOI: $10.1007 / \mathrm{s} 13320-014-0179-\mathrm{y}$

Article type: Regular 
card (DAC) will produce the random noise in the data collection process. All these make the weak signal completely be submerged in noise, which leads to great difficulties in the signal detection and the design of the processing system. Thus, it is an important part to remove noise effectively in the RDTS system, which is directly related to the sensing capacities.

In consideration of the random noise, the cumulative average algorithm is commonly used. Although the cumulative average algorithm can improve the signal to noise ratio (SNR) effectively, the time spent on one acquisition cycle will be longer if the cumulative times and the data of the whole fiber are more numerous. Thus, it is not applied in the rapidly changing temperature field.

In this paper, a wavelet transform modulus maxima de-noising method is proposed. Moreover, a simple method for determining the composition scale is presented. This method reduces the processing time because the system no longer acquires too many data to be accumulated and averaged. Experiments show that this method can eliminate the noise effectively.

\section{Theory analysis}

\subsection{Theory of wavelet transform and modulus maxima de-noising}

Wavelets are families of functions $\psi_{a, b}(t)$ generated from a single base wavelet $\psi(t)$ by dilation and translation $[7,8]$ :

$$
\psi_{a, b}(t)=\frac{1}{\sqrt{a}} \psi\left(\frac{t-b}{a}\right), \quad a>0
$$

where $a$ and $b$ are the scale and position factors, respectively. The inner products of these families with a signal $f(t)$, result in the wavelet transform of $f(t)$.

$$
W_{f}(a, b)=\int_{R} f(t) \psi_{a, b}^{*}(t) d t, \quad a>0 .
$$

$W_{f}(a, b)$ is the modulus for the projection of the signal onto the wavelet function for particular values of $(a, b)$. According to the definition of $\psi(t)$, we can get

$$
W_{f}(a, b)=f(t) *\left(a \frac{d \phi_{a}}{d t}(t)\right)=a \frac{d}{d t}\left(f * \phi_{a}\right)(t) .
$$

In the wavelet transform of $f(t)$, when $b$ belongs to the neighboring area of $\left(a_{0}, b_{0}\right)$ which is chosen randomly, we will always have

$$
\left|W_{f}\left(a_{0}, b\right)\right| \leq\left|W_{f}\left(a_{0}, b_{0}\right)\right| .
$$

Then, $W_{f}\left(a_{0}, b_{0}\right)$ is the local maximum of the wavelet modulus on scale $a_{0}$. When the wavelet function is the differential coefficient of a smooth function, the local modulus maxima are caused by the singularity of the signal and random noise. In the RDTS, the local modulus maxima will be represented if the temperature make a change.

The rule of the modulus propagating across the scales is often measured by Lipschitz exponents $L$ and can be written as

$$
\left|W_{f}(a, b)\right| \leq k a^{L}
$$

where $k$ is a constant, and $a$ is the decomposition scale. From (5), we can notice that if $L>0$, the wavelet transform modulus maxima (WTMM) will increase along with an increase in the scale $a$; If $L<$ 0 , the WTMM will decrease along with an increase in scale $a$. For the signal, there is $L>0$, and the WTMM will increase along with an increase in scale $a$; for the white noise, $L<0$, the WTMM will decrease. According to this base, the WTMM of the signal and noise can be separated over the wavelet plane to improve the SNR. The special method for obtaining the WTMM of the signal is shown as follows [9]:

(1) The WTMM in each scale is obtained in the neighboring area based on $\left|W_{f}\left(a_{0}, b\right)\right| \leq\left|W_{f}\left(a_{0}, b_{0}\right)\right|$, where the $W_{f}\left(a_{0}, b_{0}\right)$ is the local maximum of the wavelet modulus on scale $a_{0}$, and $b$ is in the neighboring area.

(2) Select a threshold value $t$ at the maximum scale $a$, remove the WTMM whose amplitude is less than $t$; otherwise retain this WTMM. In the same way, the new maximum on the other scales can also be obtained. 
(3) The WTMM of the signal on every scale is obtained by the modulus propagating difference between the signal and noise, specifically as follows: construct a neighboring area $O\left(n_{a i}, \delta_{a}\right)$ at each of the maximum positions of scale $a$ at first. $n_{a i}$ is the position of the $i$-th maximum on scale $a$, and $\delta_{a}$ is the neighboring area only connected about scale $a$. The searching area is determined again according to the spread point of $n_{a-1}$. If $n_{a i-1}^{\prime}$ is the spread point of the $n_{a i-1}$, the searching area of $n_{a i}$ is from max $\left(n_{a i-1}^{\prime}\right.$, $\left.n_{a i}-\delta_{a}\right)$ to $\min \left(n_{a i}+\delta_{\mathrm{a}}, n_{a i+1}\right)$. Retain the maxima falling on each neighboring area $O\left(n_{a i}, \delta_{a}\right)$ of the maxima on scale $a-1$ and remove the maxima outside the neighboring area. In the retained maxima on each neighboring area $O\left(n_{a i}, \delta_{a}\right)$, the maxima whose amplitudes have the same sign with the corresponding one on scale $a$ and the amplitude on scale $a-1$ less than the one on scale $a$ are stored and form the new maxima on scale $a-1$. If the maxima proposed above do not exist, the maximum of $n_{a i}$ needs to be removed. Thus, we get the new maxima on scales $a-1$ and $a$. Then, order $a=a-1$, repeat step 3 until $a-2$. In the process, if the $n_{a i}$ needs to be removed, the spread points of $n_{a i}$ on the scale which is larger than $a$ are also removed.

(4) Reserve the corresponding maximum on scale 1 in the position where the maximum exists on scale 2. Finally, reconstruct the signal using the retained modulus maxima at each scale in alternating projection algorithm and then calculate the temperature using the reconstructed signal.

\subsection{Simple method for determining the decomposition scale}

Because the WTMM amplitude of the noise decreases with an increase in the decomposition scale and that of the signal is on the contrary, the noise on the scale whose WTMM amplitude increases sharply is very low, and the signal on the following scale is dominate. This scale can be taken as the decomposition scale [10].

During the experiment, the WTMM at $30{ }^{\circ} \mathrm{C}$ (near room temperature) was used to determinate the decomposition scale in order to avoid the signal too large. If the signal is much larger than the noise, the decomposition scale will be wrong because a sharp increase in the WTMM amplitude appears prematurely. The signal was decomposed in 6 scales, and the decomposition coefficients on every scale are shown in Fig. 1.

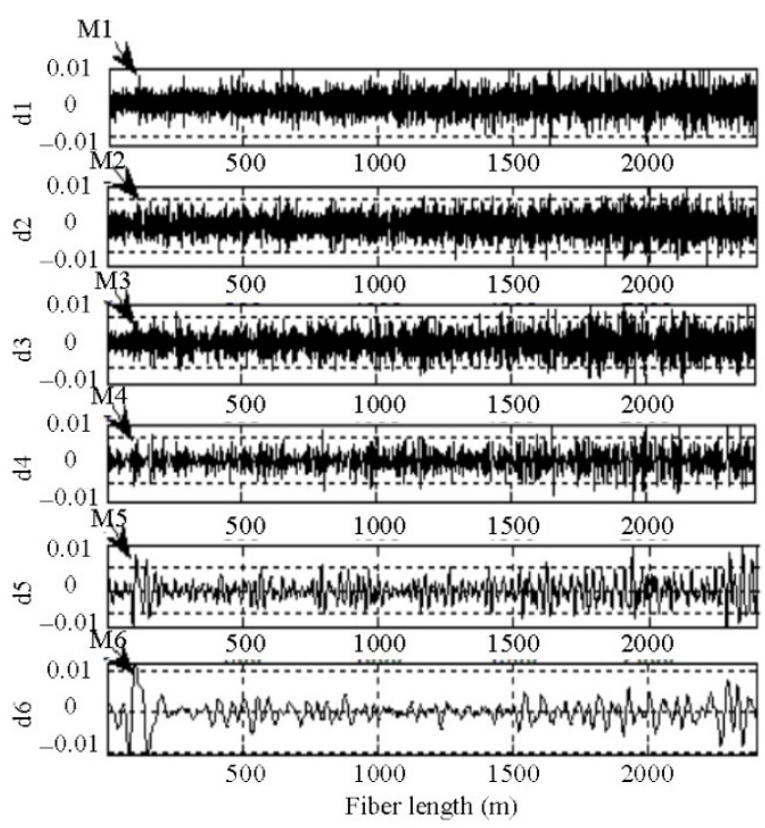

Fig. 1 Wavelet decomposition coefficients on every scale.

In Fig. 1, M1, M2, $\cdots$, M6 are expressed as the WTMM on very scale at $30{ }^{\circ} \mathrm{C}$, and M1, M2, $\cdots$, M6 are shown in Fig. 2.

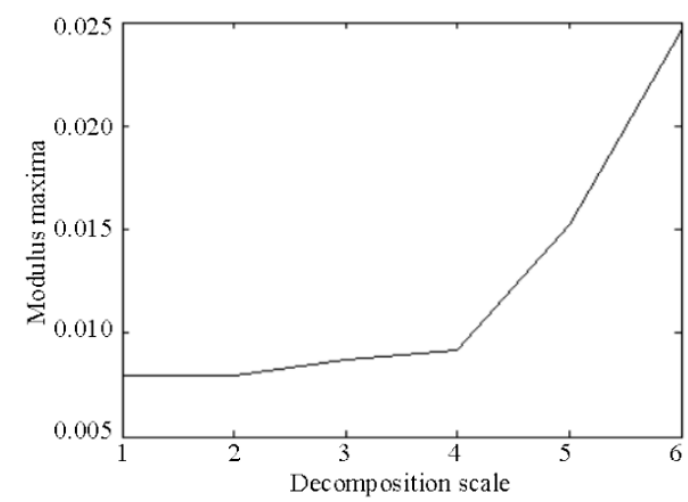

Fig. 2 WTMM on very scale.

As shown in Fig. 2, the WTMM increases with the growth of the decomposition scale, and the WTMM on scale 4 increases sharply. Thus, the decomposition scale can be defined as 4 . 


\section{Experiment}

\subsection{Experimental setup}

Experiments were conducted to verify the ability of this method to improve the accuracy of the system. The schematic diagram of the implemented experiment is shown in Fig. 3 .

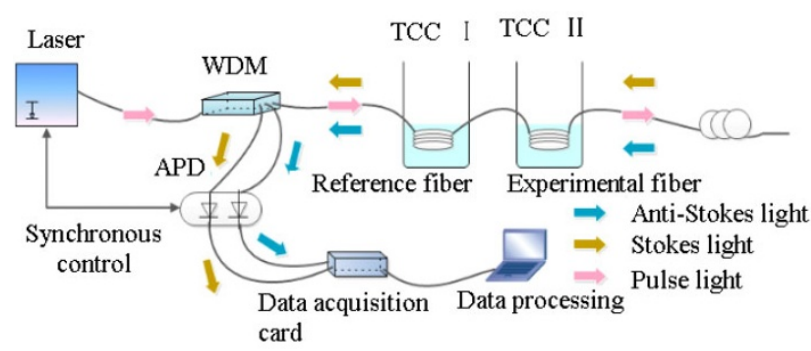

Fig. 3 Experimental setup.

The pulses with the $15-\mathrm{ns}$ width and $10-\mathrm{kHz}$ repetition rate were launched into the sensing fiber (2400-m multimode fiber) after passing through a $1 \times 3$ WDM whose isolation degree ranged from $35 \mathrm{~dB}$ up to $40 \mathrm{~dB}$. The reference fiber coils (about 50-m length) and experimental fiber (about 50-m length) were placed in temperature-controlled chambers (TCC) I and II, respectively. The pulses with the frequency $v_{0}$ generated two Raman backscattered components $\left(v_{0} \pm \Delta v\right)$ in the sensing fiber, and the Raman backscattered components then were launched into the $1 \times 3 \mathrm{WDM}$. The $1 \times 3 \mathrm{WDM}$ was employed to extract the anti-Stokes signal and Stokes signal, which were then detected and amplified by the avalanche photodiode (APD). The data were acquired by a high speed data acquisition card. At last, the data were transferred to the computer to be processed.

\subsection{Experimental results}

The experimental fiber was placed into TCC II whose temperature was set as $40{ }^{\circ} \mathrm{C}$ and $50{ }^{\circ} \mathrm{C}$, respectively. The temperatures before and after the treatment using this method at $40{ }^{\circ} \mathrm{C}$ are shown in Figs. 4 and 5 , meanwhile, those at $50{ }^{\circ} \mathrm{C}$ are show $\mathrm{n}$ in Figs. 6 and 7.
As shown in Figs. 4 and 5, the noise of the temperature after treatment is decreased comparing with that before treatment, specifically, the temperature error is decreased about $1{ }^{\circ} \mathrm{C}$ com paring with that without treatment at $40{ }^{\circ} \mathrm{C}$. Fron $\mathrm{F}$ igs. 6 and 7 , it can be seen that this result is also available for $50{ }^{\circ} \mathrm{C}$.

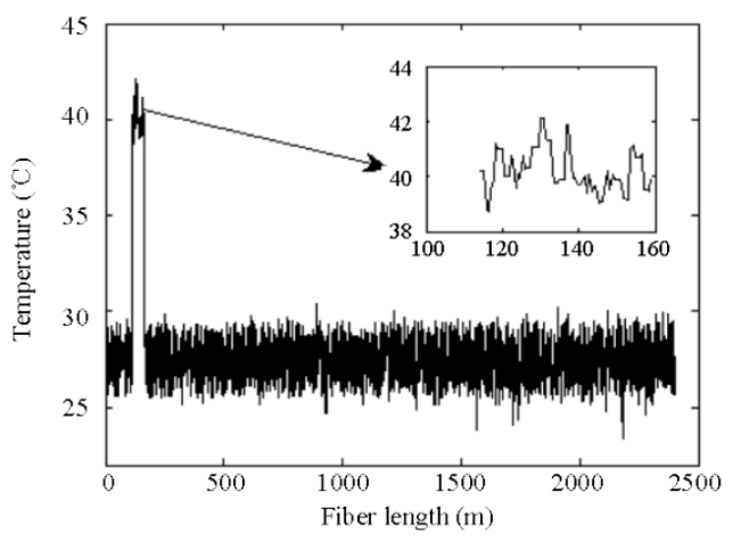

Fig. 4 Temperature before treatment at $40^{\circ} \mathrm{C}$.

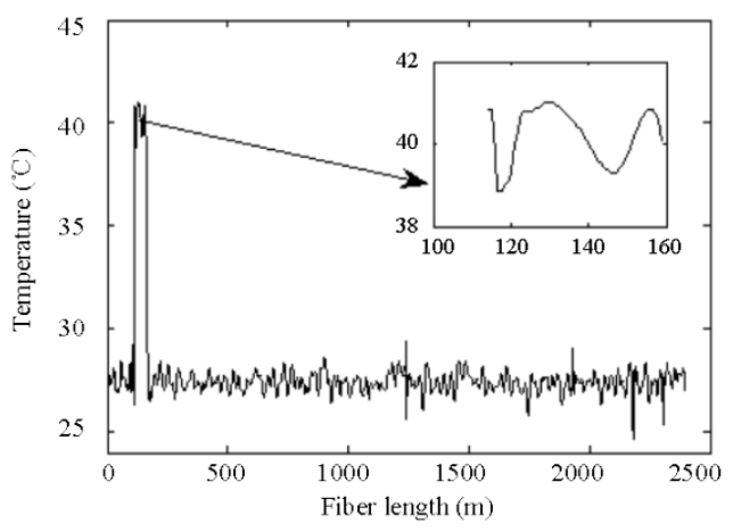

Fig. 5 Temperature after treatment at $40{ }^{\circ} \mathrm{C}$.

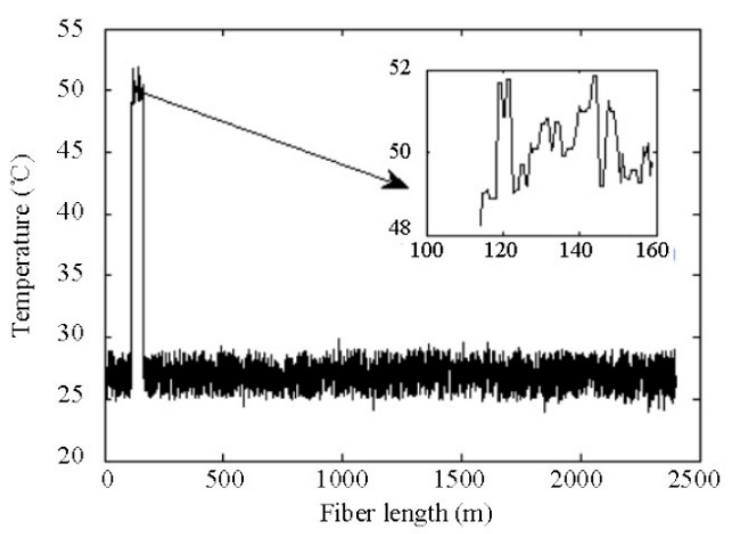

Fig. 6 Temperature before treatment at $50{ }^{\circ} \mathrm{C}$. 


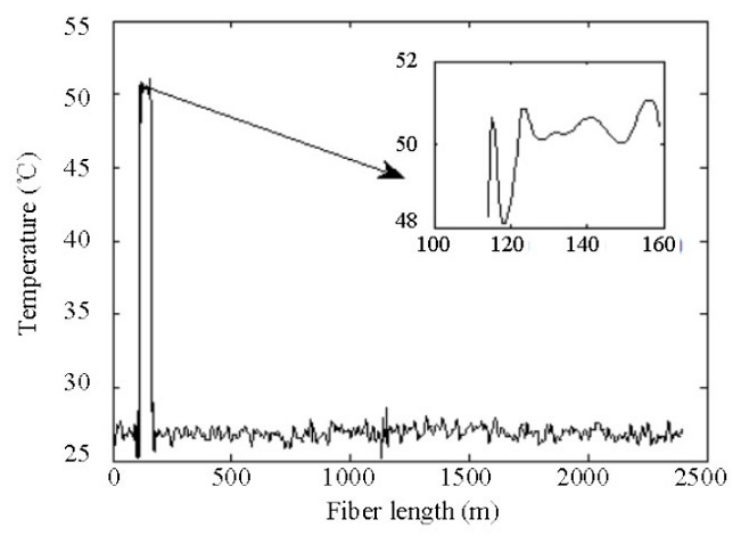

Fig. 7 Temperature after treatment at $40{ }^{\circ} \mathrm{C}$.

\section{Conclusions}

In the RDTS, the Raman signal is so weak that the signal is very difficult to be detected. The noise level in the acquired signal is very high, and the temperature error is not little. A wavelet transform modulus maxima de-noising method was proposed to decrease the temperature error. In this method, the signal WTMM were obtained by the wavelet decomposition modulus on every decomposition scale based on the modulus propagating difference between the signal and noise on every scale, and then the signal was reconstructed using the signal WTMM to eliminate the noise. Moreover, a simple method was proposed to determine the decomposition scale. Experimental results showed that the temperature error was decreased about $1{ }^{\circ} \mathrm{C}$ at $40^{\circ} \mathrm{C}$ and $50{ }^{\circ} \mathrm{C}$ comparing to the original data. Thus, this method is a powerful tool to enhance the performance of the RDTS system.

\section{Acknowledgement}

This work was supported by the Natural Science Foundation of China (60977058 \& 61307101), Independent Innovation Foundation of Shandong University (IIFSDU2012JC015) and the key technology projects of Shandong Province (2010GGX10137).

Open Access This article is distributed under the terms of the Creative Commons Attribution License which permits any use, distribution, and reproduction in any medium, provided the original author(s) and source are credited.

\section{References}

[1] S. Jiang, J. Chang, S. Zhang, Z. Wang, S. Luo, B. Sun, et al., "A method for eliminating the impact of microwave sweeper power fluctuation in BOTDA system," Photonic Sensors, 2014, 4(1): 86-91.

[2] Y. D. Gong, O. L. C. Michael, J. Z. Hao, and V. Paulose, "Extension of sensing distance in a ROTDR with an optimizediber ," Optics Communications, 2007, 280(1): 91-94.

[3] W. Wang, J. Chang, G. Lv, Z. Wang, Z. Liu, S. Luo, et al., "Wavelength dispersion analysis on fiber-optic Raman distributed temperature sensor system," Photonic Sensors, 2013, 3(3): 256-261.

[4] Z. Wang, J. Chang, S. Zhang, G. Lv, W. Wang, S. Jiang, et al., "Spatial resolution improvement of distributed Raman temperature measurement system," IEEE Sensors Journal, 2013, 13(11): 42714278.

[5] Z. Wang, S. Zhang, J. Chang, G. Lv, W. Wang, S. Jiang, et al., "Attenuation auto-correction method in Raman distributed temperature measurement system," Optical and Quantum Electronics, 2013, 45(10): 1087-1094.

[6] B. Sun, J. Chang, J. Lian, Z. Wang, G. Lv, X. Liu, et al., "Accuracy improvement of Raman distributed temperature sensors based on eliminating Rayleigh noise impact," Optics Communications, 2013, 306(1): $117-120$.

[7] Z. Qin, L. Chen, and X. Bao, "Wavelet denoising method for improving detection performance of distributed vibration sensor," IEEE Photonics Technology Letters, 2012, 24(7): 542-544.

[8] X. H. Wang, R. S. Istepanian, and Y. H. Song, "Microarray image enhancement by denoising using stationary wavelet transform," IEEE Transactions On Nanobioscience, 2003, 2(4): 184-189.

[9] B. Le, Z. Liu, and T. Gu, "Weak LFM signal dectection based on wavelet transform modulus maxima denoising and other techniques," International Journal of Wavelets, Multiresolution and Information Processing, 2010, 8(2): 313-326.

[10] J. Liu, Y. Tao, and W. Zhang, "Modulus maximum and nonlinear threshold based wavelet-denoising method," Application Research of Computers, 2008, 25(10): 3134-3135. 